\title{
CONSTRUINDO CAMINHOS, RELATANDO VIVÊNCIAS: A TRAJETÓRIA DO GRUPO DE ESTUDOS E PESQUISA EM SAÚDE
}

\author{
Suzane Beatriz Frantz Krug ${ }^{1}$, Ari Nunes Assunção' ${ }^{2}$ Leni Dias Weigelt', Luciele Sehnem ${ }^{4}$, Luciane Maria \\ Schmidt Alves ${ }^{5}$, Lívia de Almeida Faller ${ }^{6}$
}

\footnotetext{
${ }^{1}$ Doutora em Serviço Social. Professora Adjunto do Departamento de Enfermagem e Odontologia da Universidade de Santa Cruz do Sul (UNISC). Rio Grande do Sul, Brasil. E-mail: skrug@unisc.br

${ }^{2}$ Doutor em Filosofia da Enfermagem. Professora Adjunto do Departamento de Enfermagem e Odontologia da UNISC. Rio Grande do Sul, Brasil. E-mail: arias@unisc.br

${ }^{3}$ Doutora em Desenvolvimento Regional. Professor Adjunto do Departamento de Enfermagem e Odontologia da UNISC. Rio Grande do Sul, Brasil. E-mail: lenid@unisc.br

${ }^{4}$ Mestre em Medicina e Ciências da Saúde. Professora Auxiliar do Departamento de Enfermagem e Odontologia da UNISC. Rio Grande do Sul, Brasil. E-mail: lucielesehnem@unisc.br

${ }^{5}$ Mestre em Saúde Coletiva. Professora Auxiliar do Departamento de Enfermagem e Odontologia da UNISC. Rio Grande do Sul, Brasil. E-mail: lucianealves@unisc.br

${ }^{6}$ Acadêmica de Enfermagem. Bolsista PUIC/Programa UNISC de Iniciação Científica. Rio Grande do Sul, Brasil. E-mail: liviaafaller@yahoo.com.br
}

RESUMO: O Grupo de Estudo e Pesquisa em Saúde, da Universidade de Santa Cruz do Sul, tem o propósito de construir coletivamente saberes para a transformação da sociedade e aprimoramento do conhecimento em saúde. O presente artigo tem como objetivo relatar as experiências do Grupo de Estudo e Pesquisa em Saúde em seus nove anos de existência. Descreve a trajetória, atividades e projetos desenvolvidos, reflete sobre sua condução investigativa e pontua aspectos favoráveis e limitadores das práticas de pesquisa. Como delineamento metodológico utilizou-se o relato de experiência. Apresentam-se como resultados, pesquisas de enfoque qualitativo, com linha de pesquisa em saúde coletiva/saúde da família, participação na formação de discentes com estímulo para o trabalho em grupo e participação em eventos e produções cientificas. Aspectos pontuados nesta trajetória constituiram-se a partir de um grupo novo, composto de integrantes com condução investigativa recente, pertencente a uma universidade comunitária. Sem dúvida, esses fatores foram importantes na constituição da história do grupo.

DESCRITORES: Grupos de pesquisa. Saúde coletiva. Saúde da família.

\section{BUILDING PATHS, REPORTING EXPERIENCES: THE TRAJECTORY OF THE HEALTH STUDIES AND RESEARCH GROUP}

\begin{abstract}
The Health Studies and Research Group from the University of Santa Cruz do Sul, Brazil, aims to collectively construct knowledge in order to transform society, as well as improvement of health knowledge. The objective of this article is to report the experiences of the Health Studies and Research Group during its nine years of existence. It describes its trajectory, activities, and projects developed; reflects on the investigative direction; scores favorable and limiting aspects of research practices. The experience report was used as a methodological plan and resulted in research with qualitative focus, within the public health/family health line of research; participation in student training through stimulating group work; and participation in scientific events and knowledge production. The aspects scored along this trajectory were constituted from a new group, formed by members with recent investigative practice and who belong to a communitarian university. Undoubtedly, these factors have been important in establishing the group's history. DESCRIPTORS: Research groups. Public health. Family health.
\end{abstract}

\section{CONSTRUCCIÓN DE CAMINOS, RELATO DE VIVENCIAS: LA HISTORIA DEL GRUPO DE ESTUDIOS E INVESTIGACIÓN EN SALUD}

RESUMEN: El Grupo de Estudio e Investigación en Salud de la Universidad de Santa Cruz do Sul, tiene como objetivo construir colectivamente el saber para transformar la sociedad y la mejora del conocimiento en salud. En este artículo se detallan las experiencias del Grupo de Estudio e Investigación en Salud en sus nueve años de existencia. Se describe su historia, actividades y proyectos desarrollados, se reflexiona sobre su decurso investigativo, y se señalan aspectos favorables y las limitaciones de las prácticas de investigación. Como diseño metodológico se utilizó el relato de experiencia. Se presentan como resultados: investigaciones de enfoque cualitativo, con línea de investigación en salud pública/salud familiar, la formación de los estudiantes con estímulo para el trabajo en equipo, la participación en eventos y publicaciones científicas. Los aspectos señalados en esta historia se formaron a partir de un nuevo grupo, compuesto por miembros con investigaciones recientes, pertenecientes a una universidad comunitaria. Sin duda, esos factores fueron importantes en la creación de la historia del grupo.

DESCRIPTORES: Grupos de investigación. Salud colectiva. Salud de la familia. 


\section{INTRODUÇÃO}

O desenvolvimento de práticas da pesquisa em diferentes áreas tem se constituído como uma busca constante no intuito de consolidar a produção do conhecimento e de superar uma visão elitista, que coloca o pesquisar como atividade exclusiva de grandes instituições e intelectuais. ${ }^{1}$ A atividade de pesquisa é uma das molas propulsoras do avanço do conhecimento, da construção e validação de novas tecnologias e da formação e fortalecimento profissional nas diferentes áreas. ${ }^{2}$

Das diferentes áreas, a pesquisa na área de saúde coletiva tem por objetivo produzir conhecimentos que, em última instância, tornem concretas as visões e desejos relacionados à saúde da população e ajude a construir novas alternativas no sentido de prevenção das doenças, da promoção da saúde e da organização de um sistema equânime de saúde. ${ }^{3}$

Neste contexto, os grupos de pesquisa representam uma instância importante e estratégica para o desenvolvimento e consolidação da pesquisa institucional, bem como, para qualificar a inserção da universidade na realidade social e no atendimento às demandas que envolvem a produção do conhecimento científico e tecnológico. ${ }^{4}$ São definidos como conjuntos de pesquisadores, estudantes e pessoal técnico-administrativo organizados hierarquicamente em torno de uma ou, eventualmente, duas lideranças, cujo fundamento organizador dessa hierarquia deve ser a experiência, o reconhecimento e a liderança científica e/ou tecnológica. É importante que o trabalho de investigação científica esteja organizado em torno de linhas comuns de pesquisa e traduza, através dos projetos de pesquisa e da divulgação dos seus resultados, uma efetiva integração entre pesquisadores, estudantes e comunidade. ${ }^{4}$

Subjacente a essas considerações teóricas iniciais acerca da atividade de pesquisa e do processo constituinte de grupos de pesquisa, finalidade e importância, o Grupo de Estudos e Pesquisa em Saúde (GEPS) da Universidade de Santa Cruz do Sul (UNISC), propõe-se como espaço organizador e gerador de conhecimentos na área da saúde humana nas diversas circunstâncias de interação do ser humano com o meio onde vive. Além disso, intenciona tornar-se um banco de dados para projetos de atenção à saúde em consonância com as políticas públicas e acolher pesquisadores emergentes da graduação e pós-graduação. ${ }^{5}$ Constitui-se um grupo com produção correspondente à linha de pesquisa em saúde coletiva, com ênfase na saúde da família.
A Estratégia de Saúde da Família (ESF) é um modelo de atenção à saúde que o Brasil vem experimentando na tentativa de oferecer à população um atendimento adequado e satisfatório. $\mathrm{O}$ advento da ESF no Brasil, na década de 1990, reflete a tendência da valorização da família na agenda das políticas sociais, uma expansão da atenção primária na busca de incorporação de práticas preventivas, educativas e curativas mais próximas da população e, em especial, de grupos mais vulneráveis. ${ }^{6}$ Essa estratégia apresenta-se como uma nova maneira de assistir à saúde, tendo a família como centro da atenção e o planejamento das ações embasado nas reais necessidades da população e, não apenas, nas demandas espontâneas que chegam à unidade, preconizando, dessa forma, medidas e condutas preventivas. Porém, alguns municípios que adotaram a mesma não possuem como sistemática a discussão e avaliação da organização do serviço, da participação da comunidade e da relação entre os profissionais e a população, bem como a forma de organização do trabalho em equipe, a gestão do trabalho, a resolutividade das ações e a satisfação dos usuários.

Nesse contexto, alguns estudos sobre políticas públicas têm demonstrado que os formuladores de políticas operam em ambientes carregados de incertezas e que estas podem ser encontradas em situações como: limitações cognitivas derivadas da complexidade dos fenômenos sociais e das limitações dos conhecimentos das disciplinas sociais sobre a sociedade, a falta de controle e de condições para prever as contingências que podem afetar a política no futuro, a liberdade de ação aos implementadores, podendo os mesmos agir de forma arbitrária, a possibilidade de formuladores desenvolverem políticas e programas conforme suas preferências individuais ou coletivas, inclusive divergir das preferências da coletividade. ${ }^{7-8}$ A saúde pública, assim como as demais decisões políticas requerem a participação de diversos atores e acordos em vários pontos ao longo de uma cadeia de decisões tomadas por representantes em diversas arenas decisórias. ${ }^{9}$

Considerando essas ideias, o presente artigo tem como objetivo relatar as experiências do GEPS da UNISC em seus nove anos de existência. Pretende-se descrever a trajetória, as atividades e projetos desenvolvidos, refletir sobre sua condução investigativa e pontuar aspectos favoráveis e limitadores ao desenvolvimento de práticas de pesquisa.

Ao realizar a descrição dessa condução histórica e de vivências, o GEPS pretende contribuir para 
o aprimoramento de outros espaços investigativos, a partir das experiências descritas. Entende-se que a elaboração de artigos nesse formato metodológico, um relato de experiência, pode auxiliar no planejamento de ações de outros grupos de pesquisa, na qualificação das atividades propostas e no direcionamento de investigações científicas.

\section{CONSTITUIÇÃO E DIRETRIZES DO GRUPO - GEPS}

O GEPS é um dos grupos de pesquisa da UNISC, instituição universitária comunitária, situada no município de Santa Cruz do Sul-RS, localizada a $160 \mathrm{Km}$, da capital Porto Alegre. Esta instituição foi reconhecida pela Portaria $n^{\circ} 880$, de 23/06/93, com base no parecer CFE n ${ }^{\circ} 282$, de 05/05/93. Com o seu reconhecimento, acelerou-se a implantação de cursos que, em 2009, totalizavam 46 na graduação, sete programas de pós-graduação stricto sensu, sendo seis mestrados e um doutorado, e diversos cursos de pós-graduação lato sensu, nas mais diversas áreas. O caráter comunitário conduziu-a ao estágio de desenvolvimento em que se encontra, característico de uma universidade em expansão, que cresce acompanhando os avanços tecnológicos, sem descuidar-se da qualidade de ensino e do humanismo em suas ações, reforçando o compromisso com o desenvolvimento regional. ${ }^{4}$

Atualmente constituem esse grupo, cinco docentes do Departamento de Enfermagem e Odontologia - quatro enfermeiros e uma biomédica - oito discentes de enfermagem, sendo dois bolsistas do Programa UNISC de Iniciação Científica (PUIC), um bolsista do Programa Institucional de Bolsas de Iniciação Científica (PIBIC) do Conselho Nacional de Desenvolvimento Científico e Tecnológico (CNPq), cinco bolsistas voluntários do PUIC e um participante voluntário representante de órgão público, a $13^{a}$ Coordenadoria Regional de Saúde do Rio Grande do Sul (13 ${ }^{\text {a }}$ CRS/RS).

Subsidiado nessas considerações, o propósito das pesquisas desenvolvidas pelo GEPS é oferecer à enfermagem, à comunidade científica (interna e externa à universidade), às Estratégias de Saúde da Família, às autoridades voltadas às Políticas de Saúde Pública e aos usuários, informações e dados relevantes para a ampliação do conhecimento e análise da estratégia de saúde da família, além de fornecer subsídios que instrumentalizem os processos decisórios referentes ao planejamento em saúde e organização dos serviços. O grupo intenciona partilhar e construir coletivamente saberes para um propósito maior: a transformação da sociedade e aprimoramento do conhecimento em saúde e enfermagem.

No contexto da gestão dos serviços de saúde evidencia e analisa dados que fomentam discussões e investimentos na qualidade das ações de saúde e, ainda, subsidia debates nas disciplinas dos cursos da área da saúde envolvidos, aproximando a prática da teoria. A participação de docentes, alunos e instituições possibilita um olhar crítico sobre a realidade e evidencia um saber ampliado sobre saúde na formação dos profissionais.

\section{TRAJETÓRIA HISTÓRICA}

O GEPS, vinculado à UNISC, inicialmente denominado Núcleo de Estudos e Pesquisa em Saúde (NEPS), foi criado em outubro de 2001, iniciando suas atividades no ano de 2002, mediante a necessidade de existência de um grupo específico de estudos e pesquisas na área da saúde. A partir do ano de 2008, com uma organização estrutural, originou-se o GEPS, ficando, então, a cargo do núcleo, as atividades de extensão e ensino.

A ideia do grupo foi fomentada a partir da qualificação dos docentes do Departamento de Enfermagem e Odontologia, inseridos em programas de pós-graduação stricu sensu, e do crescimento e desenvolvimento de pesquisas e investigações científicas na área, sendo o grupo certificado, no mesmo ano, pelo Diretório de Grupos de Pesquisa no Brasil, pelo CNPq. Desde sua estruturação, possibilita a participação de discentes, enquanto bolsistas ou participantes voluntários em projetos institucionalizados, com ou sem financiamento de agências de fomentos. A este respeito, são inúmeros os benefícios da inserção precoce do aluno de graduação em atividades de pesquisa, entre os quais se destaca a redução do receio associado à pesquisa, o aumento da iniciativa de identificar áreas carentes de investigação, o desenvolvimento da capacidade de reconhecer os critérios de validade e confiabilidade de uma pesquisa, o comportamento de busca da superação de barreiras e obstáculos ao conhecimento, que se estende ao cuidado com o cliente, e o aprendizado do relacionamento colaborativo com os professores. ${ }^{10} \mathrm{Sob}$ este enfoque, a pesquisa oportuniza aos bolsistas participantes um processo sistemático e efetivo de apropriação e reelaboração de conhecimentos teórico-metodológicos indispensáveis para o aperfeiçoamento da investigação científica. ${ }^{11}$

No inicio de sua caminhada, o GEPS contava com uma equipe de 12 integrantes, docentes do Departamento de Enfermagem e Odontologia e 
de outros departamentos da universidade. Alguns desses integrantes pertenciam também a outros grupos de pesquisas de outras instituições de ensino, situação esta que vem ao encontro da interface científica, contribuindo para o amadurecimento e aprimoramento das discussões. Atualmente, o grupo conta com treze integrantes e desenvolve suas atividades em uma sala situada nas dependências do Serviço Integrado de Saúde (SIS) da UNISC - unidade Santa Cruz do Sul.

A missão do GEPS tem como enfoque a organização do conhecimento em saúde que represente temas aglutinadores de estudos científicos, fundamentados em tradição investigativa, de onde se originam artigos, produções bibliográficas, programas e projetos, cujos resultados se complementam. As pesquisas desenvolvidas pelo grupo caracterizam-se como de caráter social, com abordagens metodológicas do tipo qualitativo e quantitativo.

Atualmente, considera-se um grupo com produção correspondente às suas linhas de pesquisa, com ênfase na saúde coletiva e saúde da família. A linha de pesquisa denominada saúde coletiva se caracteriza por estudos de aspectos que vão além dos tratados pelas ciências naturais, envolvidos na configuração dos padrões coletivos de morbimortalidade e das formas sociais de organização do cuidado em saúde. ${ }^{12}$

\section{ATIVIDADES E VIVÊNCIAS}

A participação em um grupo de pesquisa permite a vivência de momentos, estudos e reflexões de uma riqueza, cuja dimensão, muitas vezes, é difícil definir. Emergem nesse sentido, questões como a motivação para a elaboração de um trabalho em grupo, com pessoas que, juntas, pensam em construir uma trajetória, passando pela vivência de situações fortalecedoras dessa continuidade e por experiências de limitações e superações. Relatar a trajetória do GEPS implica refletir e trazer à tona situações de satisfação e alegrias, bem como, de dificuldades.

A participação em trabalhos de grupo é uma oportunidade de compreender a necessidade de instruir-se com atores sociais, com o que acontece com os problemas na convivência cotidiana, para então, saber que dimensões do conhecimento técnico são benéficos para a vivência profissional, em sociedade, em família. ${ }^{13}$ Assim, a busca de informações in loco, nas unidades de saúde da família e nos núcleos familiares com os usuários destas unidades, traduz-se em momentos de interação, participação e valorização dos saberes dos profissionais de saúde, pesquisadores, acadêmicos e comunidade.

A pesquisa vai além da investigação; é um instrumento que proporciona aos acadêmicos a possibilidade de tornar-se um profissional autônomo, crítico e criativo, contribuindo para sua formação. Ao desenvolver atividade de pesquisa, o aluno passa a ser protagonista e corresponsável por sua aprendizagem, em que o educador ocupa um papel de facilitador desse processo. ${ }^{14}$ Assim, a introdução do estudante na iniciação científica, através do exercício da pesquisa, pode despertar para o gosto pela investigação. ${ }^{2}$

É consenso que a aprendizagem solidifica o vínculo científico em atividades acadêmicas. Portanto, o desenvolvimento de trabalhos científicos, desde os primeiros anos de formação, pode aproximar os alunos dos problemas reais da sociedade, aos quais apliquem os conhecimentos e habilidades adquiridas, de modo a formar uma atitude científica extensível à atividade profissional. Isto porque a participação em atividades de pesquisa estimula a criatividade e curiosidade e incrementa a atitude crítica dos alunos, que desenvolvem uma nova postura em relação ao saber e se tornam mais motivados para aprender..$^{15}$

A realização destas atividades complementares ao curso toma tempo e exige grande esforço dos acadêmicos, além de grande capacidade para administrar tantos compromissos de maneira responsável. Pode-se supor também que, em momentos críticos, quando as demandas das atividades se intensificam, o estudante lida com forte conflito de interesses e vive períodos de sobrecarga de trabalho e tensão para responder às solicitações. As dificuldades enfrentadas pelos estudantes que participam de pesquisas englobam a falta de tempo dos mesmos para a realização da pesquisa. A necessidade de apoio financeiro, o prejuízo no convívio com familiares e amigos pela necessidade de dedicação à pesquisa, e problemas relacionados à saúde como o estresse. ${ }^{16}$

É imprescindível relatar que a inserção de um grupo de pesquisa em uma instituição universitária e seu reconhecimento pelas instâncias internas e externas constitui-se uma etapa importante para seu fortalecimento e consolidação. A trajetória do GEPS iniciou de forma tímida, mas concisa, e vem se fortalecendo à medida que mantém contato com essas instâncias, sendo conhecido e reconhecido por elas. A divulgação ocorre através de eventos 
e também no intuito de esclarecer dúvidas e conhecer os trâmites necessários e importantes para a condução do grupo.

Os encontros dos integrantes do grupo em reuniões semanais, documentados em atas e registros específicos, constituem-se em espaço imprescindível de organização das atividades, planejamento das ações e consolidação das metas estabelecidas. Em diversas ocasiões foram necessárias modificações na condução previamente estabelecida pelo grupo, no sentido de adequar às necessidades e demandas que surgem.

A seleção, acolhimento e capacitação dos bolsistas do grupo é uma das atividades que demandam envolvimento e empenho, principalmente por parte dos docentes, no sentido de integrar ao grupo, acadêmicos que tem interesse e demonstram motivação para trilhar o caminho da investigação científica. Essas ações tornam-se gratificantes, à medida que percebe-se o crescimento e amadurecimento dos acadêmicos, e repetitivas, quando ocorre a desistência de um deles, após um período de participação no grupo. Esse fato acaba desencadeando todo um novo processo de seleção, acolhimento e capacitação, fazendo, muitas vezes, o grupo reiniciar essa trajetória, retomando atividades que já haviam sido realizadas anteriormente.

Um dos importantes fatores norteadores de uma condução investigativa científica trata dos recursos financeiros disponíveis. Desde o início de sua trajetória, o GEPS foi contemplado com recursos institucionais internos, disponibilizados pelo Departamento de Enfermagem e Odontologia, ao qual pertence, e por bolsas institucionais da UNISC aos acadêmicos. Importante considerar que o orçamento financeiro é um fator imprescindível aos grupos de pesquisa, necessitando, em muitas ocasiões, ser revisto no sentido de adequá-lo às demandas. À medida que o grupo foi se consolidando, iniciou também a participação em editais externos de órgãos de fomento à pesquisa, no intuito de oportunizar recursos humanos e financeiros para o desenvolvimento de suas atividades. Por diversas ocasiões, as tentativas não foram contempladas, fato esse que remetia o grupo a refletir a respeito, revendo seu planejamento orçamentário, estabelecendo novas diretrizes, repensando as atividades que envolveram a elaboração dos itens exigidos pelos editais. Por um lado, essa adversidade desestimulava, por outro, encorajava a seguir adiante e participar de outros editais, pois o grupo sempre considerou como premissa de seu trabalho que "o caminho se faz caminhando".
A constante participação do grupo em eventos científicos pode ser considerada uma oportunidade de socializar as atividades, conhecer experiências de outros grupos e de outros pesquisadores, identificando fragilidades e potencialidades na sua trajetória.

A aproximação com o universo dos sujeitos que integram as pesquisas, trouxe significativos e importantes avanços para a trajetória do grupo, como também enriquecimentos pessoais. As interferências de alguns fatores, como a distância geográfica entre os municípios integrantes das pesquisas e os deslocamentos para áreas íngremes e de difícil acesso, dificultaram a relação e agilidade no processo investigatório.

Portanto, considera-se que, dos contatos estabelecidos pelo GEPS com os diversos atores sociais durante sua trajetória de nove anos e também entre os próprios integrantes do grupo, emergiram histórias diversas, em espaços institucionais diferentes, mas, ao mesmo tempo, semelhantes em seus conteúdos. ${ }^{17}$

\section{Produções do grupo}

Nos últimos anos, destaca-se como produção coletiva do grupo o desenvolvimento de três pesquisas. A primeira foi desenvolvida entre os anos de 2004-2006, mediante uma metodologia quantitativa, intitulado "Indicadores de saúde da família: um estudo dos municípios circunscritos pela $13^{\mathrm{a}}$ Coordenadoria Regional de Saúde/RS". Este estudo teve como proposta a implantação, através de um grupo multiprofissional da área da saúde, de um banco de indicadores de saúde da família, a partir das informações fornecidas pela $13^{\text {a }} \mathrm{CRS/}$ RS. Pretendeu-se conhecer a realidade das ESFs, circunscritas pela $13^{\mathrm{a}} \mathrm{CRS} / \mathrm{RS}$, no que se refere às especificidades da atenção prestada, contribuindo para a organização das informações contidas no Sistema de Informações em Atenção Básica (SIAB), sendo disponibilizado à comunidade científica, aos gestores de saúde da esfera federal, estadual e municipal. Os dados foram organizados através do programas estatísticos SPSS (Statical Package for Social Science). O projeto analisou alguns indicadores de saúde, em que identificaram-se alguns índices elevados de patologias e situações de morbidade, em alguns dos 13 municípios pertencentes à $13^{\mathrm{a}}$ CRS/RS, em relação a outras Coordenadorias Regionais de Saúde, indicando que podem existir dificuldades nas ações preventivas em saúde em alguns municípios desta região. ${ }^{18}$ 
A segunda, "Saúde da família: um olhar sobre a estratégia no Município de Santa Cruz do Sul", foi desenvolvida entre os anos de 2007-2008, e teve como proposta conhecer a realidade das nove ESFs neste município, no que se refere às especificidades do seu processo de trabalho e organização das ações em saúde, investigando o processo de gestão da saúde e as repercussões no planejamento e organização dessas unidades de saúde. Objetivou-se também contribuir para o atendimento das necessidades de saúde dos usuários, através da investigação da satisfação dos mesmos em relação à assistência prestada nas unidades.

Os resultados desta pesquisa demonstraram que os usuários, em sua maioria, encontram-se satisfeitos com o atendimento prestado pelas equipes de saúde da família, enfocando o atendimento médico, odontológico, de enfermagem e das agentes de saúde, apontando como maior dificuldade as fichas para marcação de consultas e propondo algumas estratégias. Os profissionais de saúde alegaram algumas dificuldades de diálogo e de estabelecimento de um plano de ação em comum. Isso aparece no desenho de um modelo de equipe ainda segmentado por núcleo profissional, na prevalência da maior valorização do profissional médico. Na análise dos dados coletados junto aos gestores, apareceram algumas fragilidades no processo de gestão da saúde e diversidade de argumentações desses gestores sobre as questões abordadas. Essas podem ter implicações no planejamento das ações e no processo decisório. ${ }^{5}$

A terceira e atual pesquisa, desenvolvida a partir de 2009, intitulada "Saúde da família: um olhar sobre a estratégia nos municípios da $13^{\text {a }} \mathrm{Co}-$ ordenadoria Regional de Saúde/RS" trata-se de um estudo cuja proposta é conhecer a realidade dessas estratégias em onze municípios pertencentes à $13^{\mathrm{a}}$ CRS/RS, no que se refere às especificidades do seu processo de trabalho, das ações em saúde, do processo da gestão da saúde e suas repercussões no planejamento e organização dessas unidades de saúde. Pretende-se também contribuir para o atendimento das necessidades de saúde dos usuários, através da investigação da satisfação dos mesmos em relação à assistência prestada nas unidades.

As informações e divulgação dos dados da pesquisa à comunidade científica, aos profissionais envolvidos, aos usuários e aos gestores de saúde da esfera municipal ou outros segmentos constituem-se como uma das etapas do projeto, contribuindo, assim, para a qualificação da assistência à saúde nos municípios. Metodologicamente, segue-se os caminhos da investigação exploratório-descritiva, uma trajetória quanti-qualitativa, utilizando-se como instrumentos de coleta de dados entrevistas com gestores e profissionais e formulários aplicado aos usuários nas famílias. ${ }^{19}$

No que se refere às publicações, destaca-se artigos completos em periódicos como: Cinergis (UNISC), Texto \& Contexto Enfermagem, Ciência \& Saúde Coletiva e Textos \& Contextos (Porto Alegre), a publicação de capítulo do livro "Saúde no espaço escolar", trabalhos completos publicados em anais de congressos, resumos expandidos em anais de eventos, resumos publicados em anais de congressos, dentre estes, um, agraciado com a premiação de honra ao mérito, o que originou a publicação de matérias em jornais locais e entrevista para a página da Web da universidade, e ainda, apresentação de trabalhos em forma de pôster ou oralmente, e um trabalho de monografia de conclusão de curso de enfermagem. Ressalta-se que os trabalhos produzidos pelo GEPS e suas linhas de pesquisas são uma fonte inspiradora aos acadêmicos, para trabalhos científicos e monográficos.

\section{CONSIDERAÇÕES FINAIS}

As escolhas que as pessoas fazem em suas trajetórias de vida, quer no âmbito pessoal, profissional ou em outro, denotam a busca incessante por aprimoramento, crescimento, melhor entendimento de si. Essa conotação traduz a trajetória do grupo, enveredando por caminhos diversos, novos e, muitas vezes desconhecidos, porém, instigantes da curiosidade e do conhecimento científico.

Entendemos que, atualmente, o conhecimento científico não está atrelado a questões exclusivamente relacionadas à área de formação específica, mas sim, a discussões e à troca de experiências que a interação interdisciplinar e multiprofissional proporciona. A intenção de ampliar os horizontes de conhecimento, passando pelas discussões pautadas pela saúde coletiva e pela saúde da família, com ênfase na visão da integralidade e humanização na assistência à saúde, envolvendo questões sociais, contribuiu para a opção do grupo por este caminho de pesquisa.

Particularmente, em diversos momentos, as adversidades não apontavam alternativas viáveis e à medida que os fatos ocorriam, a proximidade com as discussões do campo do saber e os sentimentos de superação e determinação, estimulavam o fortalecimento do grupo de pesquisa.

Considera-se que muitos dos aspectos pon- 
tuados nessa trajetória constituiram-se a partir do espaço situacional e geográfico de sua inserção - um grupo novo, composto de integrantes com condução investigativa recente e pertencente a uma universidade comunitária com curta trajetória histórica. Sem dúvida, esses fatores são importantes na constituição da história do grupo.

\section{REFERENCIAS}

1. Camponogara S, Kirchof ALC, Gelbcke LF, Magnago TSBS. O espaço do diálogo na pesquisa em enfermagem: relato de experiência sobre a fase de coleta de dados. Texto Contexto Enferm. 2007 Out-Dez; 16(4):762-8

2. Souza SS. Ser bolsista de iniciação científica: um relato de experiência. Texto Contexto Enferm. 2003 Jul-Set; 12(3):400-3.

3. Barreto MA. Pesquisa em saúde coletiva no Brasil. Cad Saúde Pública. 2003 Mar-Abr; 19(2):354-5

4. Universidade de Santa Cruz do Sul [página na Internet]. Santa Cruz do Sul (RS): UNISC; 2009 [acesso 2009 Out 21]. Disponível em http://www. unisc.br/universidade/historico.htm.

5. Krug SBF, Assunção AN, Weiglet LD, Sehnem L, Alves LMS, Faller LA, et al. Relatório do grupo de estudos e pesquisa em saúde - GEPS.Santa Cruz so Sul (RS) Universidade de Santa Cruz do Sul; 2008.

6. Vasconcelos EM. Educação popular e a atenção à saúde da família. São Paulo (SP): Hucitec; 1999.

7. Silva PLB, Melo MAB. O processo de implementação de políticas públicas no Brasil: características e determinantes da avaliação de programas e projetos. São Paulo (SP): Núcleo de Estudos e Políticas Públicas - Unicamp; 2000.

8. Weigelt LD. Política pública de saúde: um estudo sobre o processo de implementação da descentralização/ regionalização da saúde na região do Vale do Rio Pardo-RS [tese]. Santa Cruz do Sul (RS): Universidade de Santa Cruz do Sul. Programa de Pós-Graduação em Desenvolvimento Regional; 2006.

9. Immergut EM. As regras do jogo: a lógica da política de saúde na França, na Suiça e na Suécia. Rev Bras C. Soc. 1996 Fev; (30):139-65.
10. Gomes MMF, Sanna MC. A pesquisa em enfermagem no congresso de iniciação científica de uma universidade de São Paulo. Rev Bras Enferm. 2004 Set-Out; 35(1):574-8.

11. Calazans J. Iniciação científica: construindo o pensamento crítico. São Paulo (SP): Cortez; 2002.

12. Conselho Nacional de Desenvolvimento Científico e Tecnológico [página na Internet]. Brasília (DF): CNPq; 2009 [acesso 2009 Out 01]. Disponível-em http://dgp.cnpq.br/diretorioc/fontes/detalhelinha. jsp? grupo $=5310404 J Y R F G B C \&$ seqlinha $=10$

13. Boehs AE, Stefanes C, Damiani CB, Aquino MDW. Famílias com crianças desnutridas: os desafios para trabalhar em grupo. Texto Contexto Enferm. 2005 Abr-Jun; 14(2):287-92.

14. Reibnitz KS, Prado ML. Formação do profissional crítico-criativo: a investigação como atitude de reconhecimento do mundo. Texto Contexto Enferm. 2003 Jan-Mar; 12(1):26-33.

15. Palmeira IP, Rodriguéz MB. A investigação científica no curso de enfermagem: uma análise crítica. Rev Enferm Esc Anna Nery. 2008 Mar; 12(1):68-75.

16. Senna LPC, Ferreira FP, Sigaud CHSS. A experiência de participar de pesquisa científica sob a ótica dos graduandos da escola de enfermagem da universidade de São Paulo [página na Internet]. São Paulo (SP): Universidade de São Paulo; 2008 [acesso 2009 Out 23]. Disponível em: http:// www.usp.br/ siicusp/Resumos/16Siicusp/4806.pdf

17. Krug SBF. Sofrimento no trabalho: a construção social do adoecimento de trabalhadoras da saúde [tese]. Porto Alegre (RS): Pontifícia Universidade Católica do Rio Grande do Sul. Programa de PósGraduação em Serviço Social; 2006.

18. Assunção NA, Krug SBF, Weigelt LD, Schilling AZ, Malfatti CRM, Wagner R, et al. Relatório do projeto: Indicadores de saúde da família: um estudo dos municípios circunscritos pela $13^{\text {a }}$ Coordenadoria Regional de Saúde/RS. Santa Cruz do Sul (RS): UNISC; 2006.

19. Krug SBF, Assunção AN, Weiglet LD, Sehnem L, Alves LMS, Faller LA et al. Projeto de pesquisa, saúde da família: um olhar sobre a estratégia nos município da 13a. Coordenadoria Regional de Saúde/RS. Santa Cruz do Sul (RS): UNISC; 2009. 